\title{
Pseudocapacitance of Zeolite-Templated Carbon in Organic Electrolytes
}

Khanin Nueangnoraj, ${ }^{a}$ Hirotomo Nishihara, ${ }^{\text {a, }}$ Takafumi Ishii, ${ }^{\text {a }}$ Norihisa Yamamoto,

Hiroyuki Itoi, ${ }^{\mathrm{b}}$ Raúl Berenguer, ${ }^{\mathrm{c}}$ Ramiro Ruiz-Rosas, ${ }^{\mathrm{d}}$ Diego Cazorla-Amorós, ${ }^{\mathrm{d}}$ Emilia Morallón, ${ }^{\mathrm{c}}$ Masashi Ito, ${ }^{\mathrm{e}}$ Takashi Kyotani ${ }^{\mathrm{a}}$

a Institute of Multidisciplinary Research for Advanced Materials, Tohoku University, 2-1-1 Katahira, Aoba, Sendai 980-8577, Japan

b Department of Applied Chemistry, Faculty of Engineering, Aichi Institute of Technology, 1247 Yachigusa, Yakusa, Toyota 470-0392, Japan

c Departamento de Química Inorgánica e Instituto Universitario de Materiales, Universidad de Alicante. Apdo. 99, Alicante. Spain

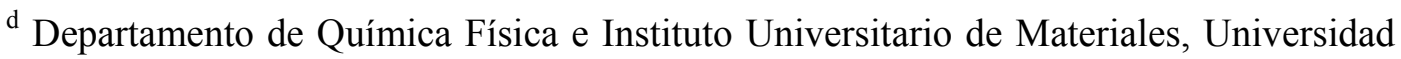
de Alicante. Apdo 99, Alicante. Spain

e Advanced Materials Laboratory, Nissan Research Center, Nissan Motor Co. Ltd., 1 Natsushima-cho, Yokosuka-shi, Kanagawa 237-8523, Japan

*Corresponding author. Tel: +81 22217 5627. Fax: +81 22217 5626. E-mail address: nisihara@tagen.tohoku.ac.jp (H. Nishihara) 


\section{Abstract}

Carbon materials and graphene-based materials, including reduced graphene oxides, can be used for supercapacitors and batteries, and they often show some amount of pseudocapacitance, which are owing to their oxygen functional groups. However, such pseudocapacitance is generally negligible in organic electrolytes and it has not attracted much attention thus far. In this work, we report a large pseudocapacitance derived from oxygen functional groups in a unique carbon material, zeolite-templated carbon (ZTC) which has significant amount of active edge sites. ZTC can be easily electrochemically oxidized in $1 \mathrm{M}$ tetraethylammonium tetrafluoroborate dissolved in propylene carbonate $\left(\mathrm{Et}_{4} \mathrm{NBF}_{4} / \mathrm{PC}\right)$, and a large amount of oxygen functional groups are introduced into ZTC as a result. The electro-oxidized ZTC shows a high specific capacitance $\left(330 \mathrm{~F} \mathrm{~g}^{-1}\right)$ mainly due to a large pseudocapacitive contribution from the oxygen functional groups in the organic electrolyte. Based on the experimental results, possible redox reactions in ZTC are proposed to be the formation of anion and cation radicals of quinones and ethers, respectively. Moreover, ZTC shows a similar pseudocapacitance also in $1 \mathrm{M}$ lithium hexafluorophosphate dissolved with a mixture of ethylene carbonate and diethyl carbonate $\left(\mathrm{LiPF}_{6} / \mathrm{EC}+\mathrm{DEC}\right)$ which is used for lithium-ion batteries and lithium-ion capacitors. 


\section{Keywords}

Electrochemical reaction, Fran, Pseudocapacitors, Graphene oxides

\section{Introduction}

Towards the development of high-energy pseudocapacitors and hybrid capacitors, various types of redox-based active materials have been investigated, e.g. conductive polymer [1,2], organic compounds [3,4,5,6,7], and metal oxides [8,9]. The conductivities of these active materials are generally not sufficient for capacitor application, and therefore, they are usually mixed with carbon materials to achieve sufficiently low inner resistance. However, this results in low mass-fraction of the redox-active materials. In addition, the redox active materials are generally not durable for long-term use, and most of them are redox-active only in aqueous electrolytes, which further limits their applications. It would be ideal if conductive carbon materials themselves could exhibit a large pseudocapacitance, especially in organic electrolytes having a wide potential window (ca. $3 \mathrm{~V})$.

We have recently reported that zeolite-templated carbon (ZTC), an ordered microporous carbon prepared by using zeolite as a hard template [10,11], has a very large amount of active edge sites, namely ca. 10 times larger than the amount of ordinary activated 
carbons. Moreover, such edge sites can be easily functionalized by a large amount of quinone groups in $\mathrm{H}_{2} \mathrm{SO}_{4}$ electrolyte $[12,13]$. The quinone groups give rise to high pseudocapacitance (ca. $500 \mathrm{~F} \mathrm{~g}^{-1}$ ) with reasonably high rate capability and good cyclability [13]. In this work, we report the introduction of redox-active oxygen functional groups to ZTC in tetraethylammonium tetrafluoroborate dissolved in propylene carbonate $\left(\mathrm{Et}_{4} \mathrm{NBF}_{4} / \mathrm{PC}\right)$. The edge sites of $\mathrm{ZTC}$ are so active that the functional groups are easily introduced even within a potential range which is too narrow for general carbon materials to be electrochemically-reacted. The oxygen functional groups thus introduced in ZTC show a large pseudocapacitance at two different potentials in the organic electrolyte. We then try to understand the origin of the pseudocapacitance. Moreover, we examine the behavior of ZTC in lithium hexafluorophosphate dissolved with a mixture of ethylene carbonate and diethyl carbonate $\left(\mathrm{LiPF}_{6} / \mathrm{EC}+\mathrm{DEC}\right)$ to see if $\mathrm{ZTC}$ exhibits a similar pseudocapacitive behavior in the different type of organic electrolyte.

\section{Experimental}

ZTC was prepared using the method reported elsewhere [14]. ZTC is characterized by a very large BET surface area of $3600 \mathrm{~m}^{2} \mathrm{~g}^{-1}$ as well as by ordered micropores with a 
uniform size of $1.2 \mathrm{~nm}$. A three-electrode cell was constructed inside a glove box (Ar atmosphere). An electrode sheet for a working electrode was prepared by mixing ZTC, PTFE, and carbon black with the weight ratio of 90:5:5. About $10 \mathrm{mg}$ of the electrode sheet was sandwiched by a Pt mesh to form the working electrode. Using the same manner, a counter electrode was also prepared, but an activated carbon fiber (Unitika, A20) was used instead of ZTC. The working electrode was isolated from a counter electrode by a cellulose separator. A reference electrode consisted of $\mathrm{Ag} / \mathrm{AgClO}$. The electrochemical characterization was carried out using the three-electrode cell with $1 \mathrm{M}$ $\mathrm{Et}_{4} \mathrm{NBF}_{4} / \mathrm{PC}$ electrolyte at $25^{\circ} \mathrm{C}$, in a fixed potential range from -1.5 to $1.0 \mathrm{~V}$ to clarify the mechanisms of electrochemical oxidation of ZTC and the appearance of the resulting pseudocapacitance. For comparison, two types of commercial activated carbons (Kansai Coke and Chemicals Co., Ltd., MSC30; Kuraray Chemical Co., Ltd., YP-50F) were used as reference. MSC30 is a $\mathrm{KOH}$-activated carbon having a large BET surface area of $2680 \mathrm{~m}^{2} \mathrm{~g}^{-1}$. YP-50F is a steam-activated carbon which is indeed used as an electrode material of commercial electric double-layer capacitors. YP-50F has a moderate BET surface area of $1700 \mathrm{~m}^{2} \mathrm{~g}^{-1}$, and is highly durable for long-term use. By the electrochemical oxidation [13], quinone-functionalized ZTC was also prepared. In this case, a working electrode was prepared in the same manner as described above, 
and ZTC was electrochemically oxidized in $1 \mathrm{M} \mathrm{H}_{2} \mathrm{SO}_{4}$ electrolyte through four scans of $\mathrm{CV}$ in a potential range of -0.1 to $0.8 \mathrm{~V}$ (vs. $\mathrm{Ag} / \mathrm{AgCl} / \mathrm{Cl}^{-}$(sat.)). Then, the working electrode was thoroughly rinsed with ultrapure water and allowed to stay in ultrapure water for $48 \mathrm{~h}$. The working electrode was then rinsed again with ethanol and dried at $150{ }^{\circ} \mathrm{C}$ under reduced pressure for $24 \mathrm{~h}$ to completely remove water and ethanol in the modified ZTC. Finally, the working electrode was characterized in $1 \mathrm{M} \mathrm{Et}_{4} \mathrm{NBF}_{4} / \mathrm{PC}$.

In order to understand the effect of the electrochemical oxidation on the carbon surface chemistry, some of electrode sheets before and after the electrochemical characterizations were subjected to the temperature-programmed desorption (TPD) analysis equipped with a mass spectrometer [15]. Besides, the oxygen functional groups before and after the electrochemical oxidation were analyzed with a FT-IR spectrometer (Shimadzu, FTIR-8900) [13]. For the TPD and FT-IR analyses, the electrode sheet taken out from the current collector was washed by acetone with sonication for $30 \mathrm{~min}$. Then, the washed sample was separated from the acetone by filtration. The sonication and filtration were repeated twice and the sample was finally dried at $80{ }^{\circ} \mathrm{C}$ under reduced pressure for $6 \mathrm{~h}$.

The stable potential range of ZTC, in which ZTC shows stable charge/discharge cycles, was also examined by the following method. First, cyclic voltammetry (CV) was 
performed for 4 cycles in a narrow potential range of $-1.5 \sim 0.5 \mathrm{~V}(\mathrm{vs} \mathrm{Ag} / \mathrm{AgClO} 4)$. Then, galvanostatic charge/discharge cycling (GC) was conducted at several different current densities. Next, the upper limit potential was increased stepwise from 0.5 to 1.3 $\mathrm{V}$, and the set of $\mathrm{CV}$ and $\mathrm{GC}$ was repeated at each of the potential ranges. Similar potential expansion towards negative potential region was done in the same manner but the upper limit potential was fixed at $0.5 \mathrm{~V}$ and the lower limit potential was expanded stepwise from -1.5 to $-2.3 \mathrm{~V}$. The stable potential range of ZTC was determined from the change of the specific capacitance or color change of the electrolyte. Then, the electrochemical performance of ZTC was examined in the potential range $(-2.0$ to 1.0 V) determined by the above experiment.

A set of electrochemical measurements was made also in $1 \mathrm{M} \mathrm{LiPF}_{6} / \mathrm{EC}+\mathrm{DEC}$. In this case, stainless mesh was used instead of Pt mesh, and Li foil was used as both reference and counter electrodes.

\section{Results and Discussion}

\subsection{Pseudocapacitance of ZTC in $1 \mathrm{M} \mathrm{Et}_{4} \mathrm{NBF}_{4} / \mathrm{PC}$}

Figure 1a shows cyclic voltammograms of $\mathrm{ZTC}$ in $1 \mathrm{M} \mathrm{Et}_{4} \mathrm{NBF}_{4} / \mathrm{PC}$. The black and red lines corresponds to the 1 st and the 4 th scans measured in -1.5 to $1.0 \mathrm{~V}$, respectively. 
For comparison, the data of two activated carbons (MSC30 and YP-50F) are also shown in Figure $1 \mathrm{~b}$ and c, respectively. In Figure 1a, a large anodic current is observed above $0.5 \mathrm{~V}$ at the initial positive-direction scan up to $1.0 \mathrm{~V}$ (black line). Then, at the subsequent negative-direction scan, a redox peak is observed around $0.4 \mathrm{~V}$, and this redox peak appears at the following cycles, as can be seen in the red line. We have recently reported very similar results of $\mathrm{ZTC}$ in an aqueous electrolyte $\left(1 \mathrm{M} \mathrm{H}_{2} \mathrm{SO}_{4}\right)$ [13]. In the previous work, we have proved that ZTC is intensively oxidized at the first positive-direction scan and a large amount of quinone-type groups are introduced, which give rise to a large pseudocapacitance. From the quite similar electrochemical response mentioned above, it is possible to expect the similar electro-oxidation of ZTC also in the present organic electrolyte as well. For reference, the 1st scan (blue line) measured in $-1.5 \mathrm{~V}$ to $0.5 \mathrm{~V}$ is also shown in Figure 1a, as a datum in which ZTC is not subjected to a severe anodic condition yet. It is noteworthy that the blue line doesn't show the redox peak around $0.4 \mathrm{~V}$. Figure 1a thus indicates that the redox active species is generated during the initial positive-direction scan above $0.5 \mathrm{~V}$. Additionally, another broad and weak redox peak can be observed at a negative potential around $-0.6 \mathrm{~V}$. Unlike the case of the peak at $0.4 \mathrm{~V}$, this peak is very weak at the 1 st negative-direction scan (black line), and becomes more intense in the 4th scan (red line). Thus, the redox 
active species corresponding to this pseudocapacitance seems to be different from the origin of the pseudocapacitance around $0.4 \mathrm{~V}$, and is gradually introduced into ZTC during the four CV cycles.

The aforementioned behavior of ZTC is totally different from those of the commercial activated carbons (Figure $1 \mathrm{~b}$ and c), whose voltammograms exhibit a typical rectangular shape based on pure electric double layer capacitive behavior regardless of the number of cycle. Since the activated carbons do not show such intense anodic current as in the case of ZTC, the anodic process seen in ZTC would be due to the unique property of this carbon, and not due to the electrochemical cell. However, it is well known that Pt, the present current collector, can catalyze the anodic decomposition of PC solvent [16]. To clarify the effect of the Pt mesh (current collector), a similar electrochemical measurement to Figure 1a was carried out by using a stainless mesh (Supporting Information Figure S1). The results are almost the same as in Figure 1a, and therefore, the introduction of the redox-active species by the anodic process is due to the nature of ZTC itself.

To investigate the origin of the pseudocapacitance, ZTC after the polarization was subjected to the TPD analysis. During the heating in a TPD run, the oxygen functional groups are decomposed to $\mathrm{CO}$ and $\mathrm{CO}_{2}$ gases at different temperatures, depending on 
their types. It is generally accepted that the decomposition of ethers, phenols, and quinone-type carbonyls results in the desorption of $\mathrm{CO}$, carboxylic groups and lactones give $\mathrm{CO}_{2}$, and acid anhydrides produce both $\mathrm{CO}$ and $\mathrm{CO}_{2}$ (molar ratio 1:1) $[17,18,19]$. The amount and the type of oxygen functional groups can therefore be estimated from the TPD patterns. The resulting $\mathrm{CO}$ and $\mathrm{CO}_{2}$ desorption profiles are shown in Figure 2, and the corresponding amount of oxygen is summarized in Table 1. It is found that the amounts of $\mathrm{CO}$ and $\mathrm{CO}_{2}$ desorption from ZTC increase upon the positive polarization up to $1.0 \mathrm{~V}$, indicating that oxygen functional groups are indeed introduced into ZTC by the electrochemical oxidation in $1 \mathrm{M} \mathrm{Et}_{4} \mathrm{NBF}_{4} / \mathrm{PC}$. In Figure 2, major enhancement can be seen in the $\mathrm{CO}$ evolution around $400-1000^{\circ} \mathrm{C}$. Since $\mathrm{CO}_{2}$ evolution is not similarly increased, the large $\mathrm{CO}$ evolution in the oxidized ZTC would be mainly derived from ethers, phenols, and/or quinone groups $[17,18,19]$. Consequently, one or some of these groups may contribute to the pseudocapacitance.

In the present system, there are the following two possibilities for oxygen source: the solvent (PC) or a trace amount of $\mathrm{H}_{2} \mathrm{O}(<30 \mathrm{ppm})$ in the electrolyte solution. To check the latter possibility, we carried out a separate electrochemical measurement by using a limited amount of electrolyte solution $(0.7 \mathrm{ml})$. In this case, the total $\mathrm{H}_{2} \mathrm{O}$ impurity possibly contained in the electrolyte solution is less than $1.2 \mu \mathrm{mol}$. The resulting CV 
pattern is shown in Figure 3, where ZTC shows a large oxidation peak as well as a large pseudocapacitance peak. Their electric charges are 2.5 and $1.4 \mathrm{C}$, corresponding to 26 and $14 \mu \mathrm{mol}$ of electrons, respectively. Since both values are much larger than the total $\mathrm{H}_{2} \mathrm{O}$ amount $(<1.2 \mu \mathrm{mol})$ in the solution, the oxygen source of the electrochemically introduced functional groups in ZTC should not be $\mathrm{H}_{2} \mathrm{O}$, but PC. It has been reported that the present solvent (PC) reacts with many compounds, such as phenol and tiophenol, to form $\mathrm{CO}_{2}$ and hydroxyalkyl derivaties including oxygen derived from PC $[16,20]$ at a high potential. This reaction occurs also on the edge site of carbon especially on the oxygen-functional groups [20]. Therefore, it can be reasonably understood that the active edge sites of ZTC easily react with PC and new functional groups containing oxygen are introduced as a result. Since the edge sites of ZTC are electrochemically more active than those of conventional porous carbons $[12,13]$, only ZTC can react with PC in Figure 1.

\subsection{Redox reaction in the negative potential range}

As discussed above, ZTC reacts with PC during the electrochemical oxidation, and a large amount of ethers, phenols, and/or quinone groups are introduced into ZTC. Since the present electrolyte includes not only oxygen but also nitrogen and boron, it is 
necessary to consider the possibility of the introduction of other functional groups including these heteroatoms which also exhibit pseudocapacitance[21,22,23]. However, we have confirmed that functional groups containing nitrogen or boron are not introduced into ZTC by the present polarization condition (see the Supporting Information, S2). Thus, the next question is which type of oxygen functional group may be responsible for the observed pseudocapacitance. We have recently reported that ZTC can be easily electro-oxidized in $\mathrm{H}_{2} \mathrm{SO}_{4}$, and a large amount of quinone groups can be introduced into ZTC with a high selectively. In addition, the quinone groups are electrochemically active and exhibit a large pseudocapacitance based on the quinone/hydroquinone redox reaction in the acidic aquous electrolyte [13]. Thus, we prepared the quinone-functionalized ZTC, and examined its electrochemical response in $1 \mathrm{M} \mathrm{Et} \mathrm{NBF}_{4} / \mathrm{PC}$. Figure 4 shows $\mathrm{CV}$ patterns of the pristine ZTC and the quinone-fuctionalized ZTC in two potential regions. First, these samples were characterized in the range of -1.5 to $0.5 \mathrm{~V}$ (solid lines in Figure 4). The quinone-functionalized ZTC exhibits a couple of broad peaks in a negative potential range, and the peaks are more intense than those of the pristine ZTC. Quinone groups would therefore correspond to the pseudocapacitance in the negative potential range. Quinones are generally known as redox-active functional groups, which can exhibit 
pseudocapacitance in organic electrolytes [24,25]. The redox potential of quinones in organic molecules varies with the molecular structure and/or with the adjacent functional groups, both of which affect the electron affinity of quinones $[25,26]$. But, most of the observed potentials stay within the range of the broad peak in Figure 4.

\subsection{Redox reaction in the positive potential range}

Figure 4 shows the appearance of pseudocapacitance around $0.4 \mathrm{~V}$ in the pristine ZTC, after it is subjected to the electrochemical oxidation up to $1.0 \mathrm{~V}$. As discussed above, the most probable origin of this pseudocapacitance is the oxygen functional groups which are introduced into the active edge sites of ZTC. On the other hand, the quinone-functionalized ZTC does not show such pseudocapacitance in the positive potential range (Figure 4). Note that this sample was fully oxidized in $\mathrm{H}_{2} \mathrm{SO}_{4}$ beforehand and most of edge sites must be already occupied by quinone groups. Therefore the quinone-functionalized ZTC was not severely oxidized in the initial positive-direction scan, compared with the pristine ZTC (Supporting Information, Figure S3) and the oxygen functional groups responsible for the positive potential pseudocapacitance were not formed as a result. Consequently, the origin of the pseudocapacitance cannot be ascribed to quinones, but it may be ethers and/or phenols. 
Figure 5 shows FT-IR spectra of ZTC before and after the polarization up to $0.7 \mathrm{~V}$. Ethers, phenols, and quinones generally give IR absorption bands at 1000-1300, 3200-3400 and $1600-1900 \mathrm{~cm}^{-1}$ due to $\mathrm{C}-\mathrm{O}, \mathrm{O}-\mathrm{H}$ and $\mathrm{C}=\mathrm{O}$ stretching modes, respectively $[27,28]$. By the polarization up to $0.7 \mathrm{~V}$, a new peak appears in the region of the $\mathrm{C}-\mathrm{O}$ stretching, suggesting that the redox peak at $0.4 \mathrm{~V}$ (Figure 1a, 3, and 4) corresponds to ethers. It has been indeed reported that PC reacts with edge sites of carbons to produce functional groups including an ether chain [20].

To our best knowledge, a furan ring is the only ether-type group which shows reversible redox reaction in this positive potential range [29]. We have previously elucidated that ZTC framework has a significant amount of carbon-pentagons, considering its curved structure [30]. It is therefore very likely that furan rings (5-membered hetero-ring) were introduced into ZTC framework by the electrochemical oxidation. We thus propose a possible redox reaction scheme of $\mathrm{ZTC}$ in $1 \mathrm{M} \mathrm{Et}_{4} \mathrm{NBF}_{4} / \mathrm{PC}$, as shown in Figure 6 . ZTC is first electrochemically oxidized above $0.4 \mathrm{~V}$ (Figure 1a) with PC, and furan-type ethers are introduced as a result. By the following cycles, in addition to the ethers, redox-active quinones are slowly introduced in ZTC. The former is reversibly oxidized/reduced by the formation of its cation radical [29], and thus shows pseudocapacitance around $0.4 \mathrm{~V}$. The latter is also reversibly oxidized/reduced by the 
formation of its anion radical [31], and shows pseudocapacitance around $-0.6 \mathrm{~V}$.

\subsection{Stable potential range of ZTC in $1 \mathrm{MEt}_{4} \mathrm{NBF}_{4} / \mathrm{PC}$}

Figure 7 shows the change of capacitance of ZTC and MSC30, when the upper (Figure

7a) or lower (Figure 7b) limit potential is expanded to a positive or negative direction, respectively. Electric double-layer capacitance is generally independent of an operating potential. Therefore, in Figure 7a, MSC30 keeps a constant capacitance (ca. $180 \mathrm{~F} \mathrm{~g}^{-1}$ ) up to $1.6 \mathrm{~V}$. At a very high potential of $1.8 \mathrm{~V}$, the capacitance is decreased probably due to the decomposition of electrolyte and the subsequent formation of polymer-like solids which cause the pore blocking [20]. This is a typical result in general porous carbons. By contrast, ZTC shows capacitance enhancement with increasing the upper limit potential up to $1.0 \mathrm{~V}$. A similar extraordinary behavior of ZTC was previously observed also in an aqueous electrolyte [13] due to the development of pseudocapacitance. However, the capacitance declines above $1.1 \mathrm{~V}$, much lower potential than the case of MSC30 (1.8 V). Probably, the active edge sites of ZTC enhance also the decomposition reaction of the electrolyte. In our previous work, it was indeed confirmed that the decomposition of electrolyte is affected by the state of the edge sites of carbon [20]. Thus, we concluded that the upper limit of the stable potential range of ZTC in $1 \mathrm{M}$ 
$\mathrm{Et}_{4} \mathrm{NBF}_{4} / \mathrm{PC}$ is $1.0 \mathrm{~V}$.

In the negative potential region (Figure 7b), the capacitance of MSC30 is stable (ca. 200

$\mathrm{F}^{-1}$ ) down to $-2.4 \mathrm{~V}$. On the other hand, ZTC shows capacitance increase down to -2.2

$\mathrm{V}$, followed by a decline at $-2.3 \mathrm{~V}$. It should be noted that the color of the electrolyte

was however turned into right brown at $-2.2 \mathrm{~V}$, indicating the degradation of the electrolyte already at this potential. Therefore, we concluded that the lower limit of the stable potential range of ZTC is $-2.0 \mathrm{~V}$.

\subsection{Rate capability and cycle stability}

The rate capability and cycle stability of ZTC are then examined in its stable potential range $(-2.0$ to $1.0 \mathrm{~V})$. By the contribution of pseudocapacitance, ZTC indeed has a higher specific capacitance (330 $\left.\mathrm{F} \mathrm{g}^{-1}\right)$ than those of YP-50F $\left(120 \mathrm{~F} \mathrm{~g}^{-1}\right)$ and MSC30 $\left(250 \mathrm{~F} \mathrm{~g}^{-1}\right)$ in $\mathrm{GC}$ measurement at $50 \mathrm{~mA} \mathrm{~g}^{-1}$. Figure $8 \mathrm{a}$ shows capacitance dependence on current density for ZTC and MSC30. Despite the contribution of pseudocapacitance, ZTC retains a high specific capacitance $\left(224 \mathrm{~F} \mathrm{~g}^{-1}\right)$ at a large current density of 2280 $\mathrm{mA} \mathrm{g}^{-1}$, and its rate performance is good to that of MSC30. In addition, the ZTC retains $94 \%$ of its initial capacitance even after 2000 cycles of charge/discharge measurements

(Figure $8 b$ ), indicating its excellent cyclability. These results prove that the redox-active 
functional groups introduced into ZTC are very stable in the conclusive potential range $(-2.0$ to $1.0 \mathrm{~V})$ and can repeat the fast redox reactions reversibly thousands of times.

\subsection{Pseudocapacitance of ZTC in $1 \mathrm{M} \mathrm{LiPF}_{6} / \mathrm{EC}+\mathrm{DEC}$}

Redox-active organic materials (e.g. molecules and polymers) are expected as a positive electrode of rechargeable batteries $[25,32,33,34,35]$, and a high redox potential is desired to achieve a high voltage operation of the batteries. Accordingly, the pseudocapacitance of ZTC at a positive potential range by furan-type ether is of interest. Since the proposed redox mechanism (Figure 6) is based on the reversible conversion of furan into its cation radical, the pseudocapacitance can be expected regardless of the type of anion. We thus examined the electrochemical behavior of ZTC in $\mathrm{LiPF}_{6} / \mathrm{EC}+\mathrm{DEC}$, which is a common organic electrolyte for lithium-ion batteries (LIBs) and lithium-ion capacitors (LICs), and it is compared with those of MSC30 and YP-50F. For each of these carbons, four CV scans were measured at first in the range of 3.0 to 4.1 V (vs. $\mathrm{Li} / \mathrm{Li}^{+}$). Then, the upper limit potential was expanded stepwise by $0.2 \mathrm{~V}$ up to 4.7 $\mathrm{V}$ with repeating four $\mathrm{CV}$ measurements at each of the potential ranges. Figure 9 shows the fourth $\mathrm{CV}$ patterns at each potential range. Like in the case shown in Figure 1a, the capacitance of ZTC is increased upon expanding the upper limit potential, and 
ZTC finally shows broad peaks in a potential range around 3.4 to $4.4 \mathrm{~V}$. Thus, the pseudocapacitance of ZTC appears in the potential range required for positive electrodes of LIBs and LICs. On the other hand, the activated carbons (MSC30 and YP-50F) never show such a pseudocapacitance in this potential range (Figure $9 \mathrm{~b}$ and c), and they show almost constant capacitance regardless of the potential range, like the cases in $1 \mathrm{M} \mathrm{Et}_{4} \mathrm{NBF}_{4} / \mathrm{PC}$ (Figure $1 \mathrm{~b}$ and c). The results shown in Figure 9 again demonstrate the highly reactive nature of ZTC which is quite unique compared to general activated carbons, and suggest the introduction of redox-active functional groups in a variety of electrolyte solutions.

\section{Conclusion}

ZTC contains a lot of highly reactive edge sites, so it can be electrochemically oxidized very easily in organic electrolytes unlike activated carbons. By the electrochemical oxidation in $1 \mathrm{M} \mathrm{Et}_{4} \mathrm{NBF}_{4} / \mathrm{PC}$, a large amount of oxygen functional groups are introduced into ZTC. The oxidized ZTC shows two different types of redox reactions in positive and negative potential regions, respectively. The former reaction could be the formation of the cation radicals of furan-type ethers, while the latter one is assigned to the anion radicals of quinone-type functional groups. Due to the significant contribution 
of pseudocapacitance, ZTC shows a high specific capacitance of $330 \mathrm{~F} / \mathrm{g}$. Moreover, despite the large pseudocapacitive contribution, its rate capability is as good as that of activated carbons, and the cycle stability is sufficiently high. Also in $1 \mathrm{M}$ $\mathrm{LiPF}_{6} / \mathrm{EC}+\mathrm{DEC}$, which is a common electrolyte for LIBs and LICs, the experimental results suggested that ZTC is electrochemically oxidized and shows a large pseudocapacitance in a positive potential range. Thus, ZTC is expected as a redox-active material for electrochemical capacitors and for LIBs and LICs as well.

\section{Acknowledgement}

This research was partially supported by the Strategic International Cooperative Program, Japan Science and Technology Agency (T.K.); a Grant-in-Aid for Scientific Research (A), 15 H01999 (T.K.); a Grant-in-Aid for Scientific Research (B), 26286020

(H.N.); and the Spanish MINECO, FEDER funds (Project MAT2013-42007-P and PRI-PIBJP-2011-0766). This research was also supported also by Nano-Macro Materials, Devices and System Research Alliance and by Network Joint Research Center for Materials and Devices. 


\section{References}

[1] P. Novak, K. Muller, K. S. V. Santhanam, O. Haas, Electrochemically active polymers for rechargeable batteries, Chem. Rev. 97 (1997) 207-281.

[2] G. A. Snook, P. Kao, A. S. Best, Conducting-polymer-based supercapacitor devices and electrodes, J. Power Sources 196 (2011) 1-12.

[3] K. Nakahara, S. Iwasa, M. Satoh, Y. Morioka, J. Iriyama, M. Suguro, E. Hasegawa, Rechargeable batteries with organic radical cathodes, Chem. Phys. Lett. 359 (2002) 351-354.

[4] M. Yao, H. Senoh, T. Sakai, T. Kiyobayashi, Redox active poly(N-vinylcarbazole) for use in rechargeable lithium batteries, J. Power Sources 202 (2012) 364-368.

[5] S. Isikli, R. Diaz, Substrate-dependent performance of supercapacitors based on an organic redox couple impregnated on carbon, J. Power Sources 206 (2012) 53-58.

[6] Y. Hanyu, I. Honma, Rechargeable quasi-solid state lithium battery with organic crystalline cathode, Sci. Rep. 2 (2012) 453.

[7] S. Isikli, M. Lecea, M. Ribagorda, M. C. Carreño, R. Díaz, Influence of quinone grafting via Friedel-Crafts reaction on carbon porous structure and supercapacitor performance, Carbon 66 (2014) 654-661.

[8] T. Brousse, M. Toupin, R. Dugas, L. Athouel, O. Crosnier, D. Belanger, Crystalline 
$\mathrm{MnO}_{2}$ as possible alternatives to amorphous compounds in electrochemical supercapacitors, J. Electrochem. Soc. 153 (2006) A2171-A2180.

[9] N. L. Wu, Nanocrystalline oxide supercapacitors, Mater. Chem. Phys. 75 (2002) 6-11.

[10] Z. X. Ma, T. Kyotani, A. Tomita, Preparation of a high surface area microporous carbon having the structural regularity of Y zeolite, Chem. Commun. (2000) 2365-2366.

[11] H. Nishihara, T. Kyotani, Templated nanocarbons for energy storage, Adv. Mater. 24 (2012) 4473-4498.

[12] R. Berenguer, H. Nishihara, H. Itoi, T. Ishii, E. Morallon, D. Cazorla-Amoros, T. Kyotani, Electrochemical generation of oxygen-containing groups in an ordered microporous zeolite-templated carbon, Carbon 54 (2013) 94-104.

[13] H. Itoi, H. Nishihara, T. Ishii, K. Nueangnoraj, R. Berenguer-Betrian, T. Kyotani, Large qseudocapacitance in quinone-functionalized zeolite-templated carbon, Bull. Chem. Soc. Jpn. 87 (2014) 250-257.

[14] H. Itoi, H. Nishihara, T. Kogure, T. Kyotani, Three-dimensionally arrayed and mutually connected 1.2-nm nanopores for high-performance electric double layer capacitor, J. Am. Chem. Soc. 133 (2011) 1165-1167.

[15] T. Ishii, S. Kashihara, Y. Hoshikawa, J.-i. Ozaki, N. Kannari, K. Takai, T. Enoki, T. 
Kyotani, A quantitative analysis of carbon edge sites and an estimation of graphene sheet size in high-temperature treated, non-porous carbons, Carbon 80 (2014) 135-145.

[16] G. Eggert, J. Heitbaum, Electrochemical reactions of propylenecarbonate and electrolytes solved therein - A DEMS study, Electrochim. Acta 31 (1986) 1443-1448.

[17] M. J. Bleda-Martinez, J. A. Macia-Agullo, D. Lozano-Castello, E. Morallon, D. Cazorla-Amoros, A. Linares-Solano, Role of surface chemistry on electric double layer capacitance of carbon materials, Carbon 43 (2005) 2677-2684.

[18] J. L. Figueiredo, M. F. R. Pereira, M. M. A. Freitas, J. J. M. Orfao, Modification of the surface chemistry of activated carbons, Carbon 37 (1999) 1379-1389.

[19] G. E. Romanos, V. Likodimos, R. R. N. Marques, T. A. Steriotis, S. K. Papageorgiou, J. L. Faria, J. L. Figueiredo, A. M. T. Silva, P. Falaras, Controlling and quantifying oxygen functionalities on hydrothermally and thermally treated single-wall carbon nanotubes, J. Phys. Chem. C 115 (2011) 8534-8546.

[20] D. Cazorla-Amorós, D. Lozano-Castelló, E. Morallón, M. J. Bleda-Martínez, A. Linares-Solano, S. Shiraishi, Measuring cycle efficiency and capacitance of chemically activated carbons in propylene carbonate, Carbon 48 (2010) 1451-1456.

[21] D. Hulicova, J. Yamashita, Y. Soneda, H. Hatori, M. Kodama, Supercapacitors prepared from melamine-based carbon, Chem. Mater. 17 (2005) 1241-1247. 
[22] T. Kwon, H. Nishihara, H. Itoi, Q. H. Yang, T. Kyotani, Enhancement mechanism of electrochemical capacitance in nitrogen-/boron-doped carbons with uniform straight nanochannels, Langmuir 25 (2009) 11961-11968.

[23] M. Kodama, J. Yamashita, Y. Soneda, H. Hatori, K. Kamegawa, Preparation and electrochemical characteristics of N-enriched carbon foam, Carbon 45 (2007) $1105-1107$.

[24] V. J. Koshy, V. Swayambunathan, N. Periasamy, A reversible redox couple in quinone-hydroquinone system in non-aqueous medium, J. Electrochem. Soc. 127 (1980) $2761-2763$.

[25] T. Nokami, T. Matsuo, Y. Inatomi, N. Hojo, T. Tsukagoshi, H. Yoshizawa, A. Shimizu, H. Kuramoto, K. Komae, H. Tsuyama, J. Yoshida, Polymer-bound pyrene-4,5,9,10-tetraone for fast-charge and -discharge lithium-ion batteries with high capacity, J. Am. Chem. Soc. 134 (2012) 19694-19700.

[26] C. Frontana, A. Vazquez-Mayagoitia, J. Garza, R. Vargas, I. Gonzalez, Substituent effect on a family of quinones in aprotic solvents: An experimental and theoretical approach, J. Phys. Chem. A 110 (2006) 9411-9419.

[27] P. E. Fanning, M. A. Vannice, A DRIFTS study of the formation of surface groups on carbon by oxidation, Carbon 31 (1993) 721-730. 
[28] U. Zielke, K. J. Huttinger, W. P. Hoffman, Surface-oxidized carbon fibers 1. Surface structure and chemistry, Carbon 34 (1996) 983-998.

[29] M. C. Pham, P. C. Lacaze, Study of the redox process of poly(2-naphthol) film using in-situ multiple internal-refrection FTIR spectroscopy, J. Electrochem. Soc. 141 (1994) 156-160.

[30] H. Nishihara, Q. H. Yang, P. X. Hou, M. Unno, S. Yamauchi, R. Saito, J. I. Paredes, A. Martinez-Alonso, J. M. D. Tascon, Y. Sato, M. Terauchi, T. Kyotani, A possible buckybowl-like structure of zeolite templated carbon, Carbon 47 (2009) 1220-1230.

[31] B. Piro, E. A. Bazzaoui, M. C. Pham, P. Novak, O. Haas, Multiple internal reflection FTIR spectroscopic (MIRFTIRS) study of the redox process of poly(5-amino-1,4-naphthoquinone) film in aqueous and organic media, Electrochim. Acta 44 (1999) 1953-1964.

[32] T. Tomai, S. Mitani, D. Komatsu, Y. Kawaguchi, I. Honma, Metal-free aqueous redox capacitor via proton rocking-chair system in an organic-based couple, Sci. Rep. 4 (2014) 3591.

[33] Z. P. Song, H. Zhan, Y. H. Zhou, Anthraquinone based polymer as high performance cathode material for rechargeable lithium batteries, Chem. Commun. (2009) 448-450. 
[34] K. M. Ismail, Z. M. Khalifa, M. A. Azzem, W. A. Badawy, Electrochemical preparation and characterization of poly(1-amino-9,10-anthraquinone) films, Electrochim. Acta 47 (2002) 1867-1873.

[35] M. Yao, H. Senoh, S.-i. Yamazaki, Z. Siroma, T. Sakai, K. Yasuda, High-capacity organic positive-electrode material based on a benzoquinone derivative for use in rechargeable lithium batteries, J. Power Sources 195 (2010) 8336-8340. 


\section{Captions for Figures}

Figure 1. CV patterns of (a) ZTC, (b) MSC30, and (c) YP-50F, measured in $1 \mathrm{M}$ $\mathrm{Et}_{4} \mathrm{NBF}_{4} / \mathrm{PC}$ at $25{ }^{\circ} \mathrm{C}$. A scan rate is $1 \mathrm{mV} \mathrm{s}^{-1}$. The 1 st and the 4 th $\mathrm{CV}$ patterns measured in a potential range from -1.5 to $1.0 \mathrm{~V}$ are expressed by black and red lines, respectively, while the 1 st $\mathrm{CV}$ pattern measured in a potential range from -1.5 to $0.5 \mathrm{~V}$ is expressed by a blue line.

Figure 2. $\mathrm{CO}$ and $\mathrm{CO}_{2}$ TPD patterns of electrode sheets including $\mathrm{ZTC}$, (a) before and (b) after polarization between -1.5 to $1.0 \mathrm{~V}$.

Figure 3. The initial voltammogram of $\mathrm{ZTC}$ in $0.7 \mathrm{ml}$ of $1 \mathrm{M} \mathrm{Et}_{4} \mathrm{NBF}_{4} / \mathrm{PC}$ between -1.5 to $1.0 \mathrm{~V}$ at $25^{\circ} \mathrm{C}$. A scan rate is $1 \mathrm{mV} \mathrm{s}^{-1}$.

Figure 4. Cyclic voltammograms (fourth scan) of the pristine ZTC (black lines) and the quinone-functionalized ZTC (red lines), measured in $1 \mathrm{M} \mathrm{Et}_{4} \mathrm{NBF}_{4} / \mathrm{PC}$ up to $0.5 \mathrm{~V}$ (solid lines), and $1.0 \mathrm{~V}$ (dotted lines). A scan rate is $1 \mathrm{mV} \mathrm{s}^{-1}$.

Figure 5. FT-IR spectra of ZTC before and after polarization up to $0.7 \mathrm{~V}$.

Figure 6. A possible redox-reaction scheme of oxygen-functionalized ZTC in $1 \mathrm{M}$ $\mathrm{Et}_{4} \mathrm{NBF}_{4} / \mathrm{PC}$.

Figure 7. Capacitance versus (a) lower or (b) upper potential range of GC measurement $\left(0.1 \mathrm{~A} \mathrm{~g}^{-1}\right)$ in $1 \mathrm{M} \mathrm{Et}_{4} \mathrm{NBF}_{4} / \mathrm{PC}$ for ZTC and MSC30. The potential range was expanded 
stepwise from $0.5 \mathrm{~V}$ to more positive range in the case of (a), and from $-1.5 \mathrm{~V}$ to more negative range in the case of (b).

Figure 8. (a) Capacitance versus current density for ZTC and MSC30, measured between -2.0 to $1.0 \mathrm{~V}$ in $1 \mathrm{M} \mathrm{Et}_{4} \mathrm{NBF}_{4} / \mathrm{PC}$. (b) Durability test of ZTC upon 2000 charge/discharge cycles, measured at $1 \mathrm{Ag}^{-1}$ in $1 \mathrm{M} \mathrm{Et}_{4} \mathrm{NBF}_{4} / \mathrm{PC}$.

Figure 9. CV patterns (fourth cycle) of (a) ZTC, (b) MSC30, and (c) YP-50F, measured in $1 \mathrm{M} \mathrm{LiPF}_{6} / \mathrm{EC}+\mathrm{DEC}$. Scan rate is $1 \mathrm{mV} \mathrm{s}^{-1}$. 


\section{Captions for Table}

Table 1. Amounts of $\mathrm{CO}, \mathrm{CO}_{2}$, and $\mathrm{H}_{2} \mathrm{O}$ evolutions and total oxygen content of the pristine ZTC electrode and the one polarized in -1.5 to $1.0 \mathrm{~V}$. 
Fig. 1
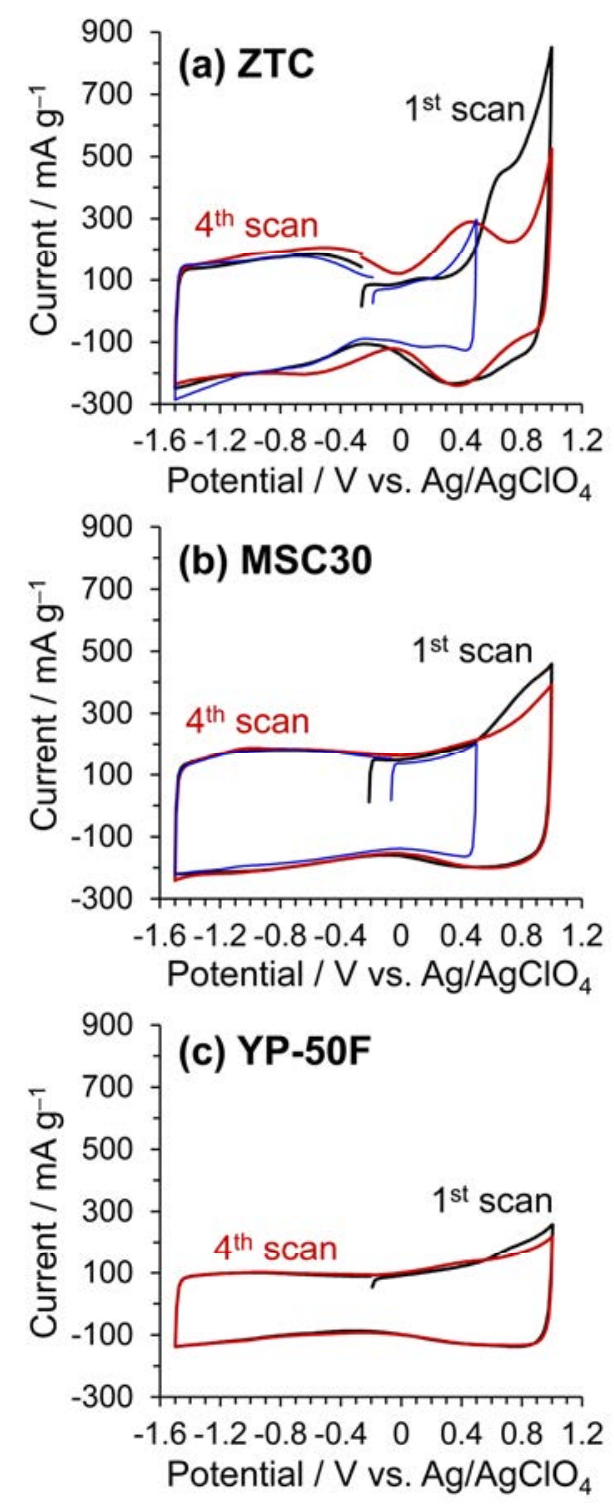
Fig. 2

(a) Pristine ZTC
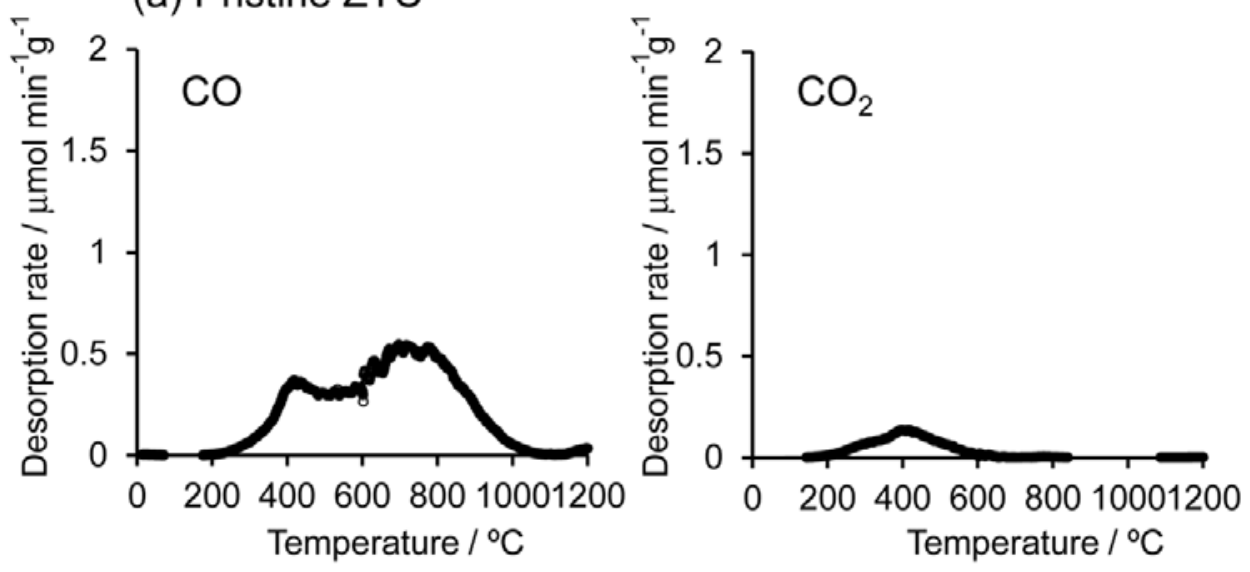

(b) ZTC (-1.5 to $1.0 \mathrm{~V})$
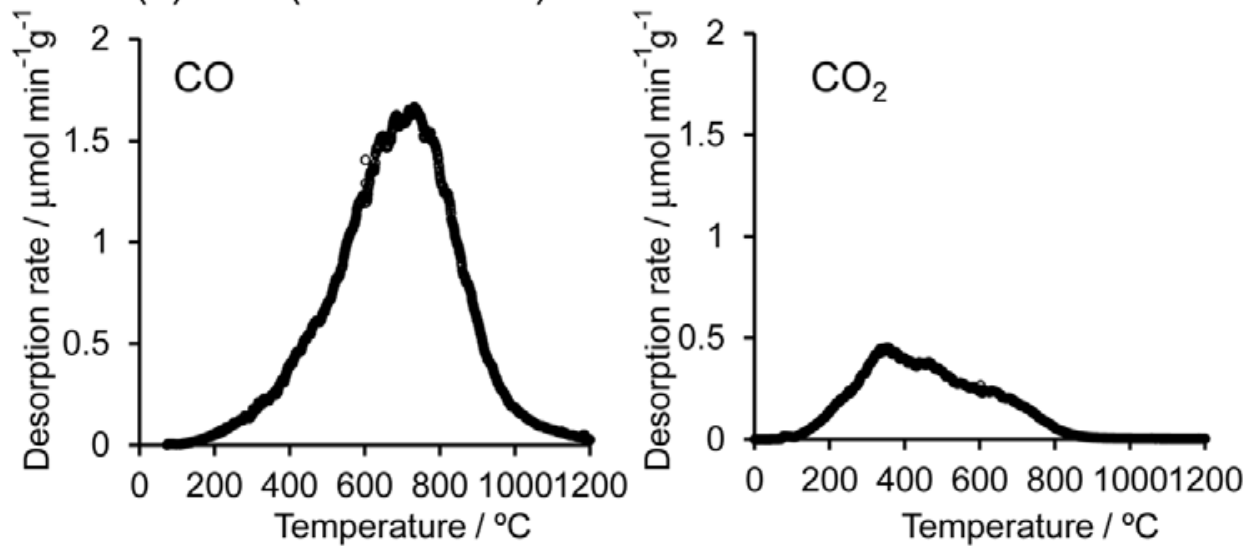
Fig. 3

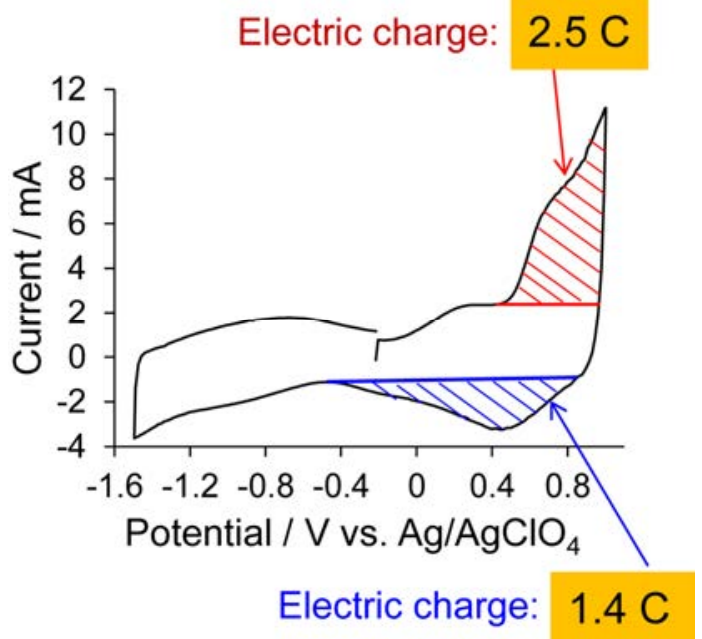


Fig. 4

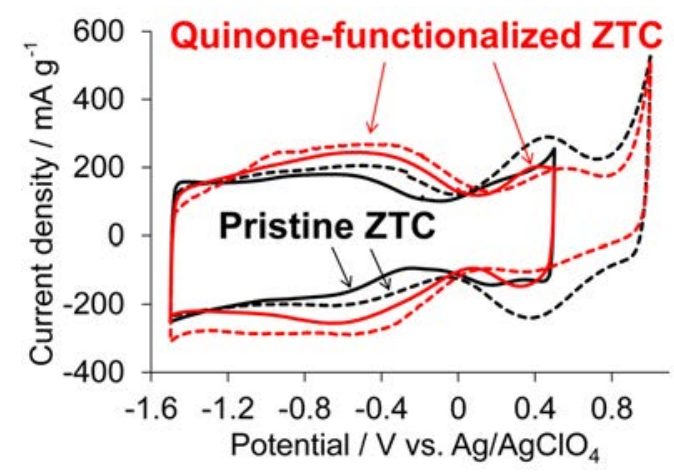


Fig. 5

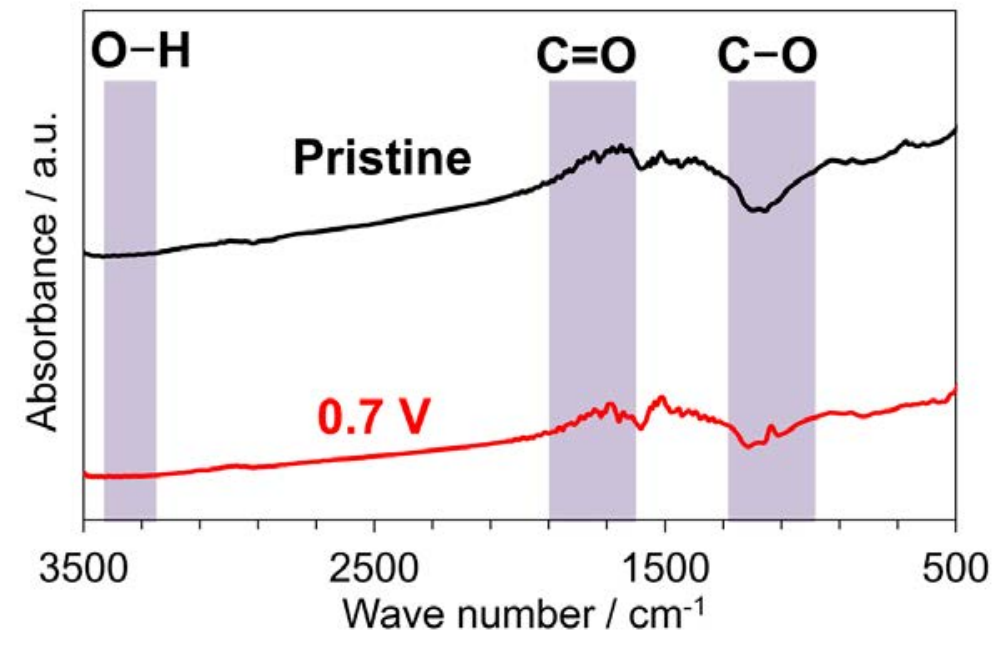


Fig. 6

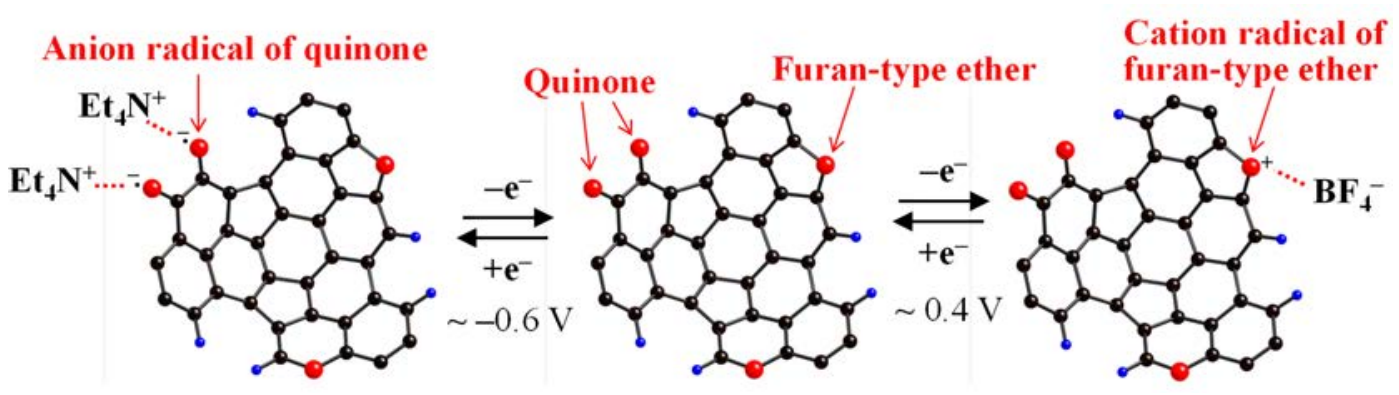


Fig. 7
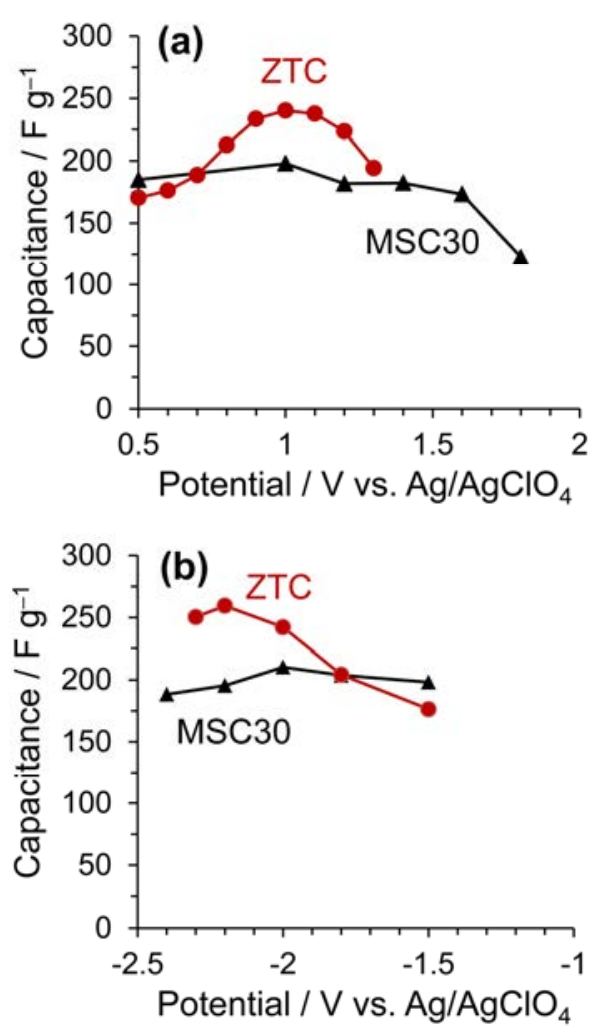
Fig. 8
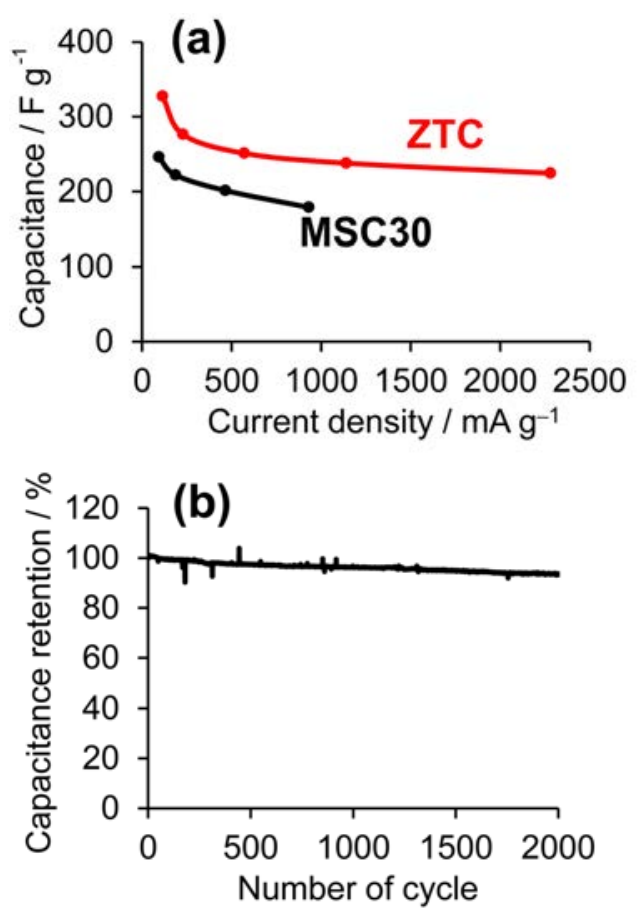
Fig. 9
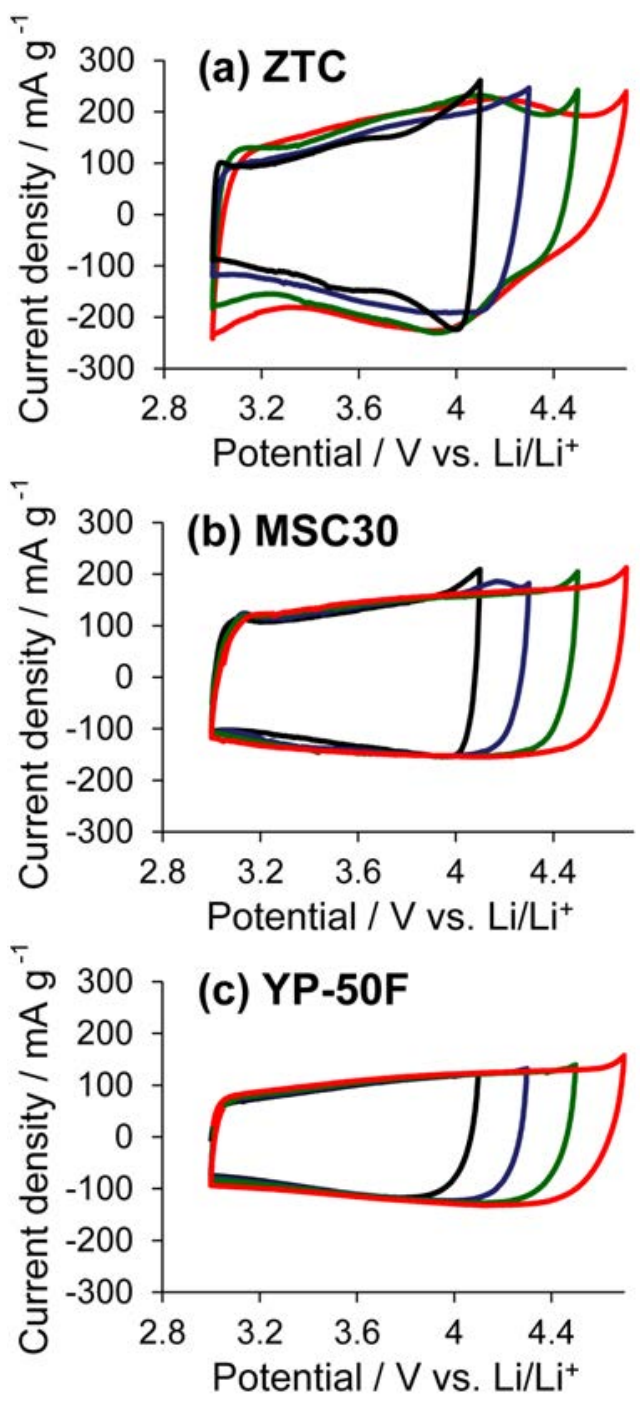
Table 1.

\begin{tabular}{ccccc}
\hline $\begin{array}{c}\text { Polarization } \\
\text { potential }(\mathrm{V})\end{array}$ & \multicolumn{3}{c}{$\begin{array}{c}\text { Amount of gas evolution } \\
\left(\mu \mathrm{mol} \mathrm{g}^{-1}\right)\end{array}$} & $\begin{array}{c}\text { Total oxygen } \\
\text { content } \\
(\mathrm{wt} \%)\end{array}$ \\
\cline { 2 - 4 } & $\mathrm{CO}$ & $\mathrm{CO}_{2}$ & $\mathrm{H}_{2} \mathrm{O}$ & 4.9 \\
\hline Pristine & 1624 & 173 & 1095 & 14.4 \\
\hline
\end{tabular}

\section{Graphical abstract}

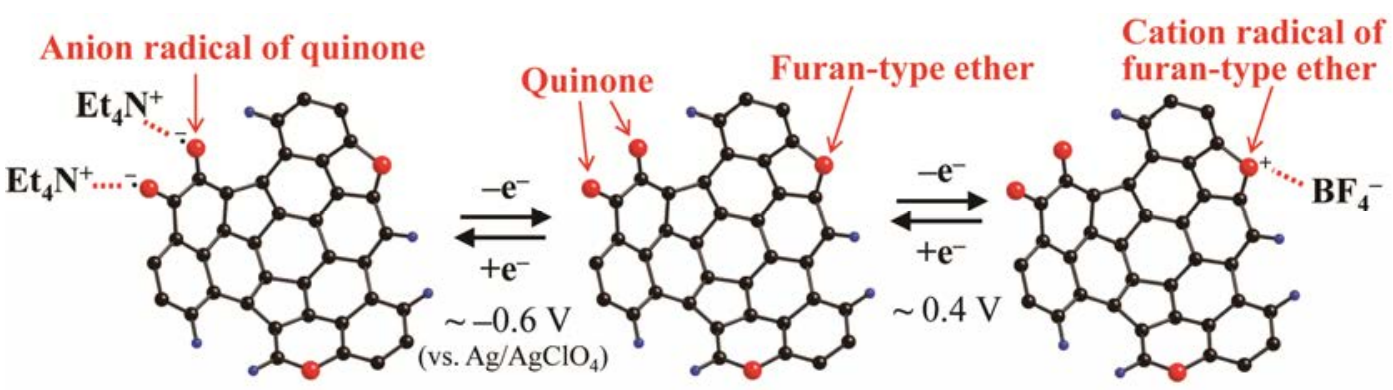

